OPEN ACCESS

Edited by:

Yongye Huang,

Northeastern University, China

Reviewed by:

Yanhua Tian,

University of Texas MD Anderson

Cancer Center, United States

Yusheng Wang,

Shanxi Provincial Cancer Hospital,

China

*Correspondence:

Guangyu Wang

Wangguangyu03@163.com

Jie Sun

suncarajie@wmu.edu.cn

${ }^{t}$ These authors have contributed equally to this work and share first authorship

Specialty section:

This article was submitted to Pharmacology of Anti-Cancer Drugs,

a section of the journal

Frontiers in Oncology

Received: 23 March 2021 Accepted: 04 May 2021

Published: 24 May 2021

Citation:

Yu Y, Zhang Z, Meng Q, Ma Y, Fan X, Sun J and Wang $G$ (2021) Comparison of the Efficacy of S-1 Plus Oxaliplatin or

Capecitabine Plus Oxaliplatin

for Six and Eight Chemotherapy

Cycles as Adjuvant Chemotherapy in

Patients With Stage II-III Gastric

Cancer After D2 Resection.

Front. Oncol. 11:684627.

doi: 10.3389/fonc.2021.684627

\section{Comparison of the Efficacy of S-1 Plus Oxaliplatin or Capecitabine Plus Oxaliplatin for Six and Eight Chemotherapy Cycles as Adjuvant Chemotherapy in Patients With Stage II-III Gastric Cancer After D2 Resection}

\author{
Yuanyuan $\mathrm{Yu}^{1,2 \dagger}$, Zicheng Zhang ${ }^{2 \dagger}$, Qianhao Meng ${ }^{1 \dagger}$, Yue $\mathrm{Ma}^{1}$, Xiaona Fan ${ }^{1}$, Jie Sun ${ }^{2 *}$ \\ and Guangyu Wang ${ }^{1 *}$

\begin{abstract}
${ }^{1}$ Department of Gastrointestinal Medical Oncology, Harbin Medical University Cancer Hospital, Harbin, China, ${ }^{2}$ School of Biomedical Engineering, School of Ophthalmology \& Optometry and Eye Hospital, Wenzhou Medical University, Wenzhou, China
\end{abstract}

Objective: To compare the efficacy of adjuvant chemotherapy with six or eight cycles of S-1 plus oxaliplatin (SOX) or Capecitabine plus oxaliplatin (XELOX) after D2 resection of GC.

Design and participants: We collected 470 cases of patients with TNM stage II and III GC who underwent D2 gastrectomy in the Harbin Medical University Cancer Hospital from January 2007 to December 2017 and received six or eight cycles of SOX or XELOX regimen. This study was designed to evaluate the prognosis of patients receiving six or eight cycles of SOX or XELOX chemotherapy and identify the appropriate number of chemotherapy cycles.

Results: Among the 470 study participants [340 (72.3\%) males; median age, 50 years (range, 24-76 years)], 355 and 115 received XELOX or SOX regimen chemotherapy, respectively. The number of 152 patients included in this study who received 6 and 8 cycles of chemotherapy in stage II and stage III without considering chemotherapy regimens were 125 and 27. The median DFS was, respectively, 14.9 months and 26.8 months ( $P=0.08)$, the median OS was, respectively, 30.2 months and 30.8 months $(P=$ $0.5)$, the difference was not statistically significant. Comprehensive survival analysis of XELOX and SOX group showed no significant difference for DFS $(P=0.29)$ and OS $(P=$ 0.61). The total number of stage III GC patients who received six and eight cycles of chemotherapy was 92 and 19, respectively. The median DFS of patients who received six and eight cycles of chemotherapy was 14.6 and 23.2 months $(P=0.3)$, respectively. The median OS of patients who received six and eight cycles of chemotherapy was 26 and 30.6 months $(P=0.9)$, respectively. Comprehensive analysis of DFS $(P=0.73)$ and OS $(\mathrm{P}=0.6)$ shows no difference between the XELOX group SOX groups. Subgroup analysis 
revealed significant differences in the gender $(P=0.05)$ and histological classification $(P<$ 0.05) distribution.

\begin{abstract}
Conclusion: Regardless of the XELOX regimen or the SOX regimen, similar survival benefits are observed in patients receiving six or eight chemotherapy cycles irrespective of the regimen used. The XELOX and SOX regimens are well tolerated in patients undergoing D2 resection of GC.
\end{abstract}

Keywords: adjuvant chemotherapy, gastric cancer, chemotherapy cycles, S-1 plus oxaliplatin, capecitabine plus oxaliplatin

\section{INTRODUCTION}

Gastric cancer (GC) is the fifth most common cancer worldwide and the fourth leading cause of cancer-related death (1). GC disproportionally affects males, with the rate of affected males being almost twice that of affected females. In 2020, there will be an estimated 1.09 million new cases of GC worldwide and about 769,000 death (1). About $49.3 \%$ of new cancer cases and $58.3 \%$ of cancer deaths occur in Asia. In China, there were about 679,000 new cases of GC and 498,000 GC-related deaths in 2015, making makes GC the second to only lung cancer in terms of morbidity and mortality (2). In China, about $80 \%$ of patients with GC are at an advanced stage at the time of diagnosis, and the 5-year survival rate is less than $30 \%$ (3). Therefore, it is important to improve the prognosis of patients with stage II and stage III GC after surgery.

Postoperative adjuvant chemotherapy has become a routine treatment for patients with GC. Indications for adjuvant chemotherapy after resectable GC are: D2 gastrectomy and no preoperative treatment for postoperative patients with pathological stage II and III advanced GC. D2 gastrectomy is based on resectable GC. Four extensive clinical studies, the ACTS-GC, CLASSIC, JACCORGC-07 and ARTIST studies, have confirmed the value of postoperative adjuvant chemotherapy. The Japanese ACTS-GC trial confirmed that S-1 single-agent postoperative adjuvant chemotherapy could significantly improve the 5-year survival rate after D2 gastrectomy for locally advanced GC (4). However, this result has not been verified in other studies, and it is unclear whether patients with stage III GC can benefit from S-1 single-drug adjuvant chemotherapy. In response to this, the CLASSIC study, a randomized, open, parallel-controlled phase III clinical study involving patients from South Korea, China Mainland, and Taiwan, showed that GC patients who received XELOX adjuvant chemotherapy had a significantly higher 5-year disease-free survival (DFS) rate than did those who had surgery alone $(68 \%$ vs. $53 \%$; $\mathrm{HR}=0.58 ; 95 \% \mathrm{CI}: 0.47-0.72 ; \mathrm{P}<0.0001)$, and the overall survival (OS) rate was also significantly improved $(78 \%$ vs. $69 \%$; $\mathrm{HR}=0.66 ; 95 \% \mathrm{CI}: 0.51-0.85 ; \mathrm{P}=0.0015)$. These data confirm that XELOX regimen adjuvant chemotherapy can significantly reduce the risk of postoperative recurrence, and the benefits of prolonging DFS can then be translated into prolonging the OS of patients. As the first chemotherapy regimen validated by evidence-based medicine in the Chinese population, the classic study showed that the XELOX regimen is suitable for postoperative adjuvant chemotherapy for patients with stage II and II GC in China. It also further confirmed that postoperative adjuvant chemotherapy could play an essential role in treating locally advanced GC $(5,6)$. The Japanese JACCROGC-07 study is a randomized controlled study designed to evaluate the efficacy of S-1 combined with docetaxel in adjuvant treatment after surgery. The 3-year recurrence-free survival (RFS) of the S-1 combined with the docetaxel group was $7 \%$ higher than that of the control group. The 3-year RFS was significantly better in the treatment group than in the control group ( $65.9 \%$ vs. $49.6 \%, \mathrm{HR}=0.632,99 \% \mathrm{CI}$ : $0.400-0.998, \mathrm{P}=0.0007$ ), and $\mathrm{S}-1$ combined with docetaxel is recommended as the new standard for adjuvant treatment after D2 gastrectomy in patients with stage III GC (7). The Korean ARTIST study compared postoperative radiotherapy and chemotherapy after D2 surgery to postoperative adjuvant chemotherapy (Capecitabine combined with cisplatin). The results indicate that the DFS and OS of the two groups are similar. Subsequently, the ARTIST-II study, enrolling patients with GC and positive lymph nodes after D2, was designed. The results showed that compared with S-1 single-drug, the SOX regimen alone and in combination with radiotherapy can significantly prolong DFS. However, SOX regimen combined with radiotherapy did not improve survival when compared to SOX regimen alone (8-10). The results of these clinical studies indicate that surgery is the only possible cure for GC, and postoperative adjuvant chemotherapy is the main way to achieve long-term survival for patients with GC.

Based on the efficacy and safety of chemotherapy, the level I recommended choices for postoperative adjuvant chemotherapy for Chinese is XELOX and SOX. S-1 is a fluorouracil derivative, and the main components are tegafur, gemerazine, and otixiracet potassium. The curative effect of $S-1$ is equivalent to that of capecitabine. However, S-1 is superior to capecitabine in increasing the concentration and time of 5-FU in tumor tissue and blood and reducing side effects, including hand-foot syndrome $(11,12)$. In 2019, the RESOLVE study showed that eight cycles of SOX adjuvant chemotherapy after D2 radical resection of GC is not inferior to XELOX (13). Currently, eight chemotherapy cycles are recommended for patients with stage II and stage III GC, irrespective of whether they are undergoing the XELOX or SOX regimen. Individual differences in patients' tolerance to chemotherapy drugs mean that some patients 
cannot tolerate the toxicity of chemotherapy drugs, leading to the early termination of chemotherapy. Therefore, for this group of patients, we aimed to compare the prognosis of patients who received six or eight cycles of XELOX or SOX adjuvant chemotherapy after radical resection of GC. These insights will allow practitioners to choose a suitable chemotherapy cycle for patients to avoid the occurrence of chemotherapy-related adverse reactions. Moreover, this data provides valuable evidence supporting the need for patients with advanced GC to receive standardized and individualized treatment, which can prolong their lives, improve their quality of life, and reduce the social burden on their families.

\section{METHOD}

\section{Study Design}

This is a retrospective study. In the real world, the proportion of people who can complete eight cycles of standard postoperative adjuvant chemotherapy for gastric cancer is not large. Most patients have completed six cycles of adjuvant chemotherapy. This research aims to compare whether six cycles are not inferior to eight cycles in the real world. We conducted a three-phase study. First, we compared the DFS and OS of patients with TNM stage II and III GC that received six or eight chemotherapy cycles without considering the chemotherapy regimen. Second, we compared the DFS and OS of patients who received six and eight chemotherapy cycles of XELOX and SOX. In the third step of the study design, the DFS and OS of patients with TNM stage III GC and 6 or 8 chemotherapy cycles were compared with and without considering the chemotherapy regimen.

\section{Inclusion Criteria}

Data were collected from patients who were pathologically diagnosed with GC (GC)/gastric junction adenocarcinoma (GEJC) and had undergone D2 radical resection and postoperative adjuvant chemotherapy at the Harbin Medical University Cancer Hospital from January 2007 to December 2017. This study was approved by the Ethics Committee of the Harbin Medical University Cancer Hospital. Patient data were confidential, and the study complied with the Declaration of Helsinki.

Case inclusion criteria were (1): preoperative endoscopic biopsy or postoperative pathological diagnosis of GC/GEJC; (2) having undergone D2 radical operation; (3) postoperative pathological staging of stage II and stage III disease based on the American Joint Committee on Cancer (AJCC) TNM staging (8th edition); (4) postoperative hematology and imaging evaluation of patients show that they meet the criteria of postoperative adjuvant chemotherapy, and tolerate chemotherapy drugs; and (5) their postoperative adjuvant chemotherapy is XELOX or SOX.

Case exclusion criteria were: (1) patient received chemotherapy regimens other than S-1, SOX, or XELOX after D2 radical surgery for GC; (2) patient was unable to complete the specified adjuvant chemotherapy cycle as required for any reason; (3) for any reason, the standard chemotherapy dose was reduced by more than 30\%; (4) patients received neoadjuvant therapy; and (5) patients with distant metastasis or relapse within 6 months during operation and after the operation, were excluded.

\section{Treatment Criteria}

XELOX treatment regimen was: oxaliplatin $130 \mathrm{mg} / \mathrm{m} 2$ (intravenous drip) on day 1 , repeated every three weeks and capecitabine $1000 \mathrm{mg} / \mathrm{m} 2$ (oral) on days 1-14, twice a day, repeated every three weeks. SOX treatment regimen was: oxaliplatin $130 \mathrm{mg} / \mathrm{m} 2$ (intravenous drip) on day 1, repeated every three weeks and S-1 $40 \mathrm{mg} / \mathrm{m} 2$ (oral) on days 1-14, twice a day, repeated every three weeks. The two treatment groups were subject to 6 or 8 cycles of chemotherapy. During chemotherapy, symptomatic and supportive treatments including antiemetic, liver protection, and stomach protection were administered. Bultrasound, CT and other imaging examinations were performed every three cycles to evaluate the treatment effect.

\section{Research Targets}

DFS is defined as the time from the date of GC D2 resection to the occurrence of recurrence, metastasis, or death. The OS is the time from the date of GC D2 resection to death due to any cause. The primary study endpoint was 5-year OS, and the secondary study endpoint was 3-year DFS. All patients were followed up for at least five years.

\section{Statistical Methods}

Clinicopathologic characteristics of patients receiving six and eight chemotherapy cycles were compared using the Chi-square test. The univariate Cox regression analysis to measure the association between treatment regimens and prognosis. The DFS and OS survival curves were drawn using the Kaplan-Meier method, and the log-rank test was used for comparison. $\mathrm{P}<0.05$ was considered statistically significant. All statistical analysis was performed with $\mathrm{R}$ Statistical Software (version 4.0.3).

\section{RESULT}

\section{Patient Clinical Characteristics}

The patient's condition and tumor characteristics are shown in Table 1. This is a real-world study of patients with GC (stage IIIII) who underwent D2 gastrectomy and received adjuvant chemotherapy with SOX or XELOX regimen in Harbin Medical University Cancer Hospital from January 2007 to December 2017. We conducted a retrospective study of 470 patients who underwent D2 resection and received postoperative adjuvant chemotherapy at our hospital and completed at least four cycles of adjuvant chemotherapy with either XELOX or SOX regimens. There were 159 patients available for analysis of DFS and 203 patients available for analysis of OS. Postoperative pathological staging was stage II or stage III in 369 patients, of which 290 patients received six cycles of chemotherapy and 79 patients received eight cycles of chemotherapy. Following the administration of chemotherapy, 51 patients were excluded 
based on the exclusion criteria. A total of 152 patients were included in the analysis according to the exclusion criteria.

The clinical characteristics of the 6-cycle and 8-cycle chemotherapy groups were similar. There were no significant differences in Lauren classification, tumor location, TNM staging, or WHO grade $(\mathrm{P}>0.05)$. There was an obvious difference between these two groups in age distribution, with most patients under 65 years of age, but this difference was not statistically significant $(\mathrm{P}=0.063)$. Obvious differences in gender distribution $(\mathrm{P}=0.05)$ and histological classification $(\mathrm{P}=0.48)$ were observed (Table $\mathbf{1})$.

\section{Survival Outcome for Stage II-III GC Patients Treated With Six or Eight Chemotherapy Cycles}

All patients were followed up for at least 5 years. We conducted a comprehensive analysis of the data for patients with stage II and stage III, irrespective of whether they underwent XELOX or SOX regimens. Patient survival was then compared between groups. The number of patients receiving six and eight cycles of chemotherapy was 125 and 27, respectively. Patients receiving six or eight chemotherapy cycles had similar rates of DFS and OS. The Kaplan-Meier method was used for survival analysis and to draw DFS and OS survival curves. Median DFS time of patients receiving six and eight cycles of chemotherapy was 14.9 and 26.8 months $(\mathrm{P}=$

TABLE 1 | Clinical characteristics of patients with gastric cancer after D2 resection enrolled in this study.

\begin{tabular}{|c|c|c|c|c|}
\hline Clinical characteristics & $\begin{array}{l}\text { Cycle } 6 \\
(n=290)\end{array}$ & $\begin{array}{c}\text { Cycle } 8 \\
(n=79)\end{array}$ & $\begin{array}{c}\mathrm{X}- \\
\text { squared }\end{array}$ & $\begin{array}{c}\mathrm{p}- \\
\text { value }^{\mathrm{a}}\end{array}$ \\
\hline \multicolumn{5}{|l|}{ Age } \\
\hline$<=65$ & 248 & 60 & 3.455 & 0.063 \\
\hline$>65$ & 42 & 19 & & \\
\hline \multicolumn{5}{|l|}{ Gender } \\
\hline Male & 196 & 63 & 3.826 & 0.05 \\
\hline Female & 94 & 16 & & \\
\hline \multicolumn{5}{|l|}{ Lauren } \\
\hline Intestinal type & 60 & 14 & 1.678 & 0.642 \\
\hline Diffuse type & 73 & 24 & & \\
\hline Mixed type & 43 & 14 & & \\
\hline Unknown & 114 & 27 & & \\
\hline \multicolumn{5}{|l|}{ Tumor_size } \\
\hline Cardia & 9 & 2 & 1.540 & 0.463 \\
\hline $\begin{array}{l}\text { Gastric body or Whole } \\
\text { stomach }\end{array}$ & 82 & 28 & & \\
\hline Gastric antrum & 199 & 49 & & \\
\hline \multicolumn{5}{|l|}{ TNM } \\
\hline$\|$ & 127 & 34 & $<0.001$ & 1 \\
\hline III & 163 & 45 & & \\
\hline \multicolumn{5}{|l|}{ WHO_grade } \\
\hline Adenocarcinoma & 155 & 42 & 0.111 & 0.991 \\
\hline Signet ring cell carcinoma & 19 & 5 & & \\
\hline Low adhesion carcinoma & 19 & 6 & & \\
\hline Mixed cancer & 97 & 26 & & \\
\hline \multicolumn{5}{|l|}{ Histological classification } \\
\hline Poorly differentiated & 142 & 27 & 7.914 & 0.048 \\
\hline Moderately differentiated & 124 & 47 & & \\
\hline Well differentiated & 10 & 1 & & \\
\hline Undifferentiated & 14 & 5 & & \\
\hline
\end{tabular}

${ }^{a} p$-value was derived from the Chi-square test.
0.08), respectively. Median OS time of patients receiving six and eight cycles of chemotherapy was 30.2 and 30.8 months $(P=0.5)$ (Figures 1A, B). The number of patients with stage II and stage III GC receiving six and eight cycles of XELOX regimen chemotherapy were 109 and 18, respectively. Median DFS time of patients receiving six and eight cycles of chemotherapy was 16 and 27 months $(\mathrm{P}=0.07)$, respectively. The median OS times of patients receiving six and eight cycles of chemotherapy, respectively, were 30 and 31.9 months $(\mathrm{P}=0.9$. The number of patients receiving six and eight cycles of SOX regimen chemotherapy was 16 and 9, respectively. The median DFS times in these patient groups were 13.7 and 24.2 months $(P=0.6)$, respectively. The median OS times in these patient groups were 21.5 and 24.5 months $(\mathrm{P}=0.5)$, respectively. Comprehensive analysis of DFS $(\mathrm{P}=0.29)$ and OS $(\mathrm{P}=$ 0.61 ) between the XELOX and SOX groups revealed no statistical difference (Figures 1C, D). In patients receiving six chemotherapy cycles, DFS and OS did not differ between those receiving XELOX and SOX regimens (DFS, $\mathrm{P}=0.97$ and $\mathrm{OS}, \mathrm{P}=0.83$ ) (Figures 2A, B). In patients receiving eight chemotherapy cycles, DFS and OS did not differ between those receiving the XELOX and the SOX regimens (DFS, $\mathrm{P}=0.49$ and $\mathrm{OS}, \mathrm{P}=0.084$ ) (Figures 2C, D).

Among all patients with stage III GC, 92 and 19 received six and eight chemotherapy cycles, respectively. Overall DFS time in patients receiving six and eight cycles of chemotherapy were 14.6 and 23.2 months $(\mathrm{P}=0.3)$, respectively. The median OS times in patients receiving six and eight cycles of chemotherapy were 26 and 30.6 months $(\mathrm{P}=0.9)$, respectively (Figures $\mathbf{3 A}, \mathbf{B}$ ).

In patients with stage III GC, 77 and 11 patients received six and eight cycles of the XELOX regimen chemotherapy, respectively. The median DFS times for patients receiving six and eight cycles of chemotherapy were 14.7 and 23.2 months $(\mathrm{P}=$ $0.6)$, respectively. The median OS times for patients receiving six and eight cycles of chemotherapy, 28 and 30.7 months $(P=0.6)$, respectively. the difference was not statistically significant. The number of people receiving six and eight cycles of SOX regimen chemotherapy was 15 and 8, respectively. The median DFS times for patients receiving six and eight cycles of chemotherapy were 13.7 and 26.8 months $(\mathrm{P}=0.6)$, respectively. The median OS times for patients receiving six and eight cycles of chemotherapy were 21.5 and 24.5 months $(\mathrm{P}=0.6)$, respectively. No differences in DFS $(\mathrm{P}=0.73)$ and $\mathrm{OS}(\mathrm{P}=0.6)$ were observed between the XELOX and SOX groups (Figures 3C, D). In patients receiving six chemotherapy cycles, DFS and OS did not significantly differ between the two treatment regimens (DFS, $\mathrm{P}=0.7$ and $\mathrm{OS}, \mathrm{P}=0.37$ ) (Figures $4 \mathrm{~A}, \mathrm{~B}$ ). In patients receiving eight chemotherapy cycles, DSF and OS did not differ between the two treatment groups (DFS, $\mathrm{P}=0.35$ and OS, $\mathrm{P}=0.25$ ) (Figures 4C, D).

Results of the univariate Cox regression suggested that there is no difference in survival between patients receiving six or eight chemotherapy cycles in either of the treatment regimens examined $(\mathrm{P}>0.05)$ (Figures 5A-D). Patients receiving eight cycles of XELOX regimen chemotherapy appeared to have better OS than did those receiving eight cycles of SOX or XELOX regimen chemotherapy, but this difference was not statistically significant (OS: HR, 0.46; $\mathrm{P}=0.086$ ) (Figure 5B). 
A

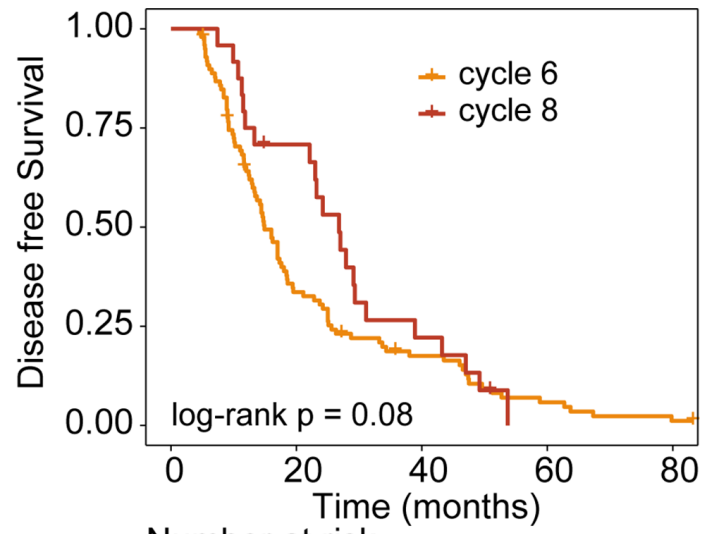

Number at risk

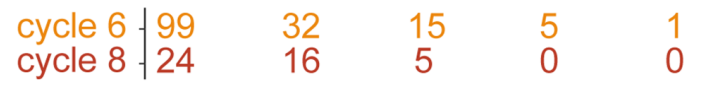

C

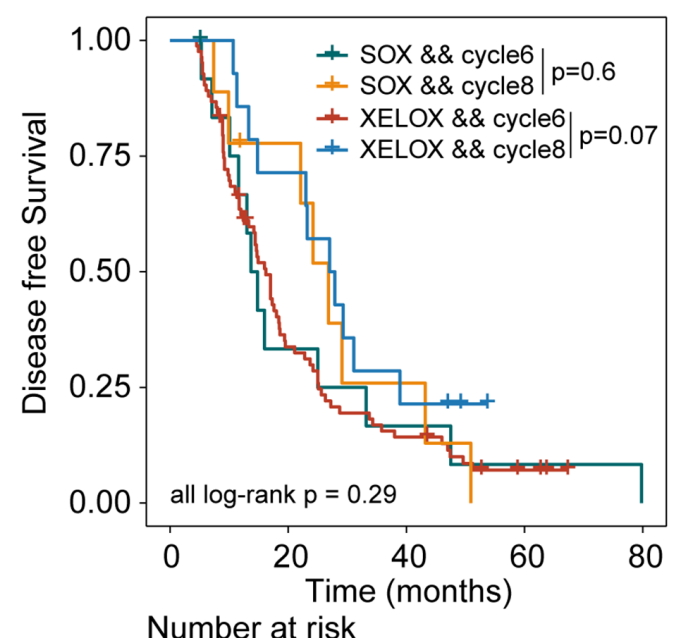

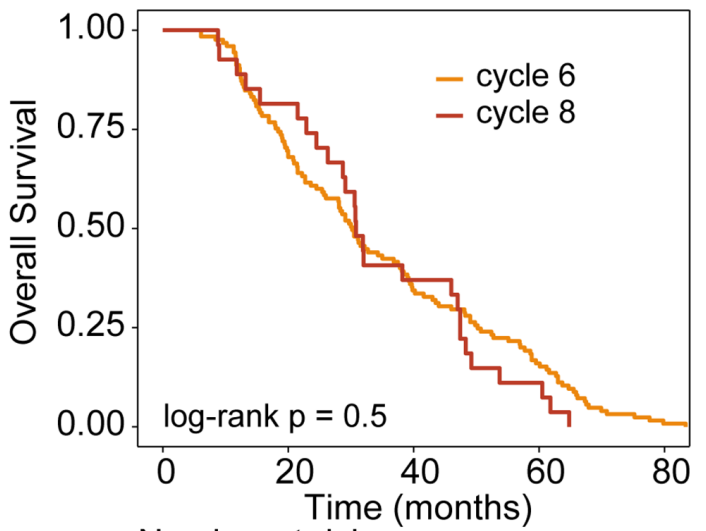

Number at risk

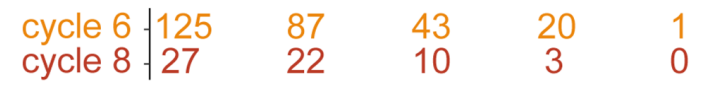

D

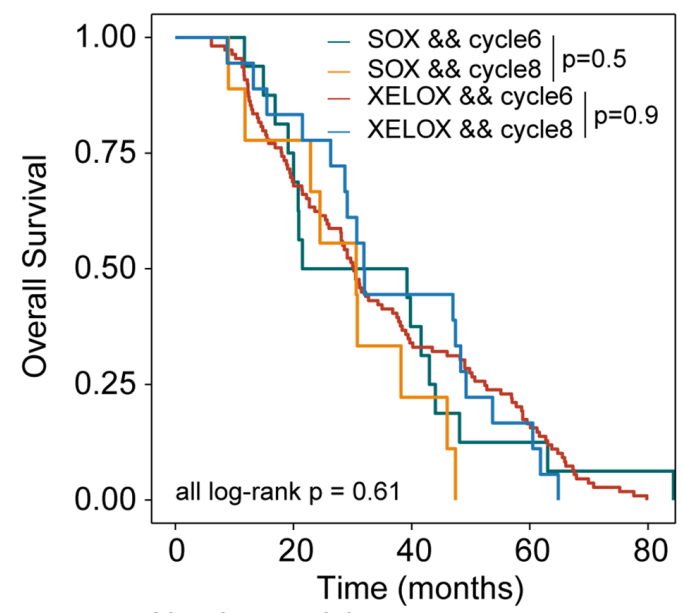

Number at risk

\begin{tabular}{|c|c|c|c|c|}
\hline SOX \&\& cycle6 & -16 & 12 & 6 & 2 \\
\hline SOX \&\& cycle8 & 9 & 7 & 2 & 0 \\
\hline XELOX \&\& cycle6 & -109 & 75 & 37 & 18 \\
\hline XELOX \&\& cycle8 & -18 & 15 & 8 & 3 \\
\hline
\end{tabular}

FIGURE 1 | Kaplan-Meier survival curves for disease-free survival (DFS) and overall survival (OS). DFS (A) and OS (B) analyses for stage II and stage III patients who received six or eight cycles of chemotherapy, irrespective of whether they underwent XELOX or SOX regimens. DFS (C) and OS (D) analyses for stage II and stage II patients who received six or eight cycles of chemotherapy, taking the specific regimen into account. XELOX, Capecitabine plus oxaliplatin; SOX, S-1 plus oxaliplatin.

\section{Subgroup Analysis}

Stratification by gender, age, Lauren classification, tumor location, TNM staging, WHO grade, and histological classification revealed similar DFS results for patients receiving six and eight cycles of chemotherapy (Figure 6A; P > 0.05). However, a significant difference was observed in DFS in patients classified as poorly differentiated histologically $(\mathrm{P}=0.034)$, suggesting that six cycles of chemotherapy for patients with GC histologically classified as poorly differentiated should be sufficient. Stratification by gender, age, Lauren classification, tumor location, TNM staging, WHO grade, and histological classification revealed similar that OS for all patients irrespective of whether they received six or eight cycles of chemotherapy (Figure 6B; P>0.05).

\section{DISCUSSION}

GC is a highly invasive and highly heterogeneous disease. The survival rate of locally advanced or metastatic disease of GC has not been significantly improved. It is still a serious global health problem. GC passes through the lymphatic system, blood and 
A

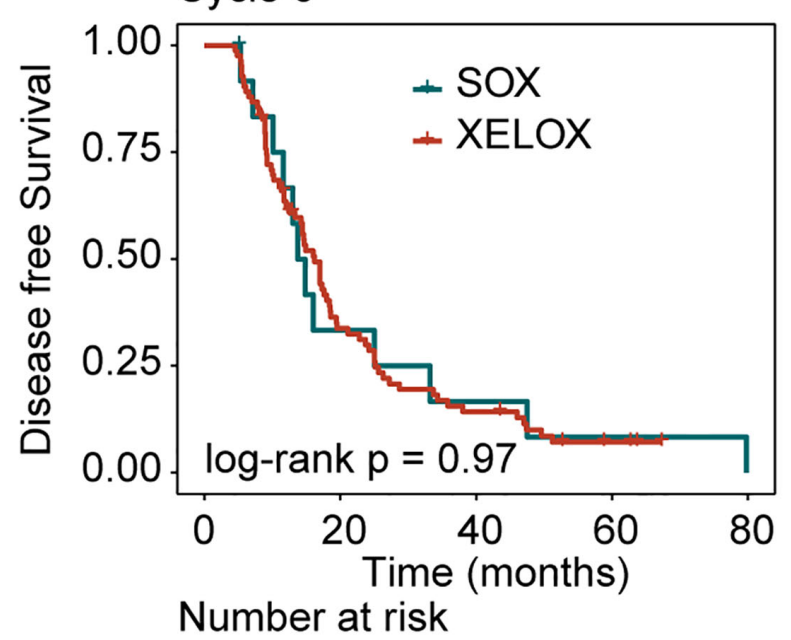

B

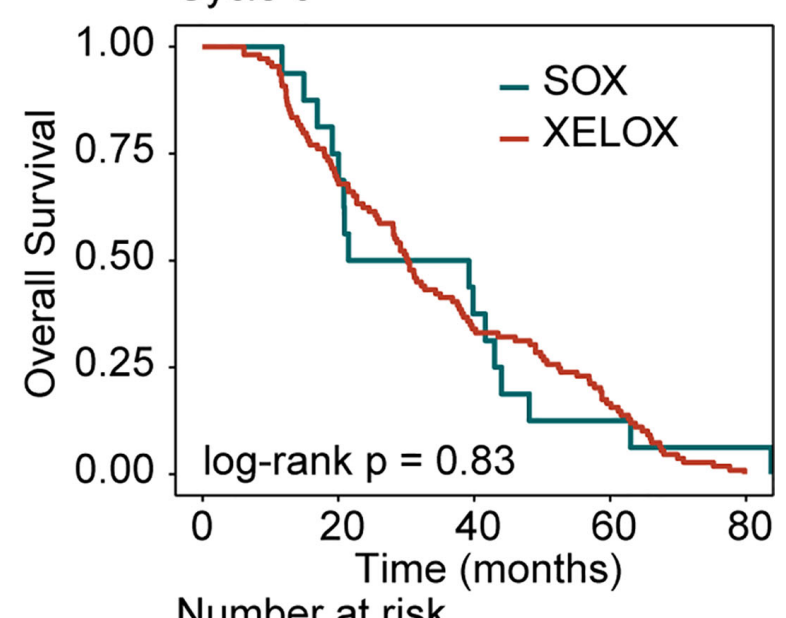

\begin{tabular}{|c|c|c|c|}
\hline sox +13 & 4 & 2 & 1 \\
\hline KELOX - 83 & 26 & 11 & 3 \\
\hline
\end{tabular}

C

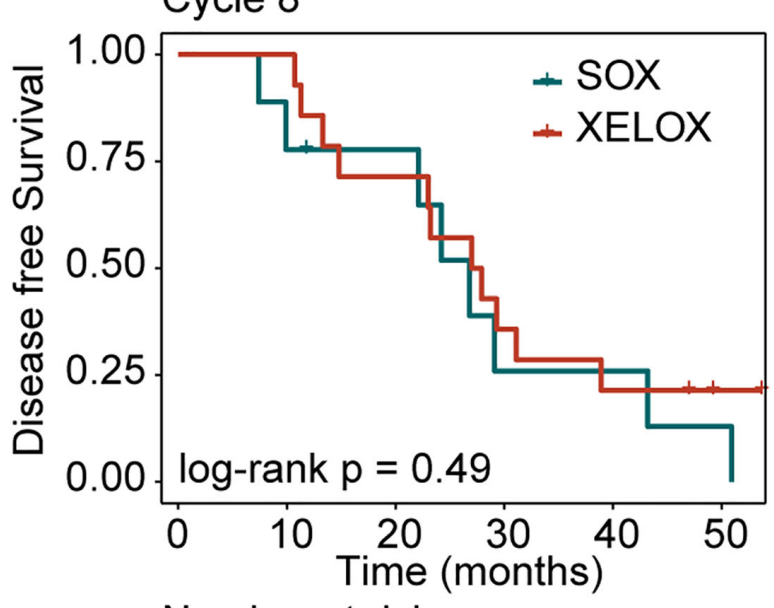

Number at risk

$\begin{array}{rccccc}\text { SOX }+9 & 7 & 6 & 2 & 2 & 1 \\ \text { XELOX }-14 & 14 & 10 & 5 & 3 & 1\end{array}$

\begin{tabular}{|c|c|c|c|c|}
\hline sox & 16 & 12 & 6 & 2 \\
\hline ELOX & 109 & 75 & 37 & 18 \\
\hline
\end{tabular}

D Cycle 8

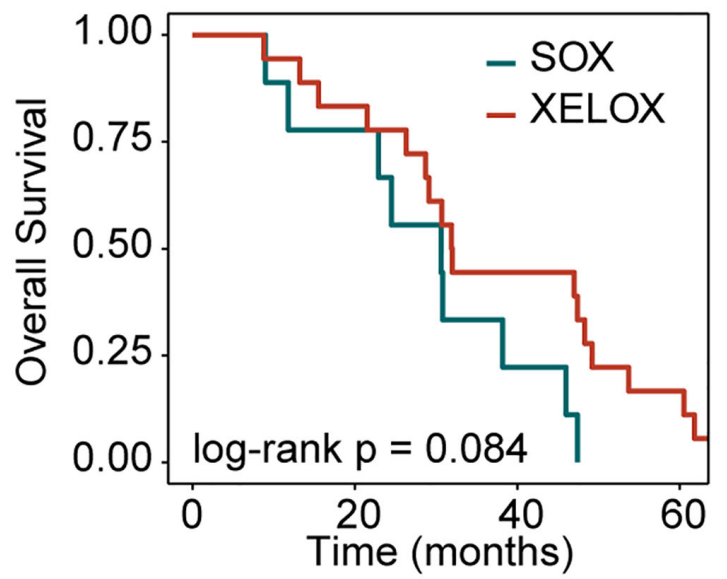

Number at risk

$\begin{array}{rrrr}\text { SOX }-9 & 7 & 2 & 0 \\ \text { XELOX }-18 & 15 & 8 & 3\end{array}$

FIGURE 2 | Kaplan-Meier survival curves for disease-free survival (DFS) and overall survival (OS). DFS (A) and OS (B) analyses for the XELOX and SOX regimens for patients with stage II and stage III gastric cancer receiving six cycles of chemotherapy. DFS (C) and OS (D) analyses for the XELOX and SOX regimens for patients with stage II and stage III gastric cancer receiving eight cycles of chemotherapy. XELOX, Capecitabine plus oxaliplatin; SOX, S-1 plus oxaliplatin.

peritoneum in the early stage. Spread, recurrence after surgery is common, about $40 \%$ of patients relapse within 2 years after surgery (14-16). In order to reduce the rate of local recurrence and metastasis of GC and prolong the survival time of patients, we routinely perform postoperative adjuvant chemotherapy for patients. SOX regimen and XELOX regimen are the first-line treatment options for advanced GC, reducing cancer recurrence, improving the survival rate, and reducing the occurrence of adverse reactions, so that the survival of patients with advanced GC has obvious benefits (17-21). In recent years, a number of large randomized clinical studies have also confirmed the status of the two regimens in adjuvant chemotherapy after GC surgery. The purpose of our research is to compare the efficacy of patients receiving 6, and 8 cycles of SOX and XELOX adjuvant chemotherapy after D2 radical resection of $\mathrm{GC}$, and to compare the prognosis of patients receiving different 
A

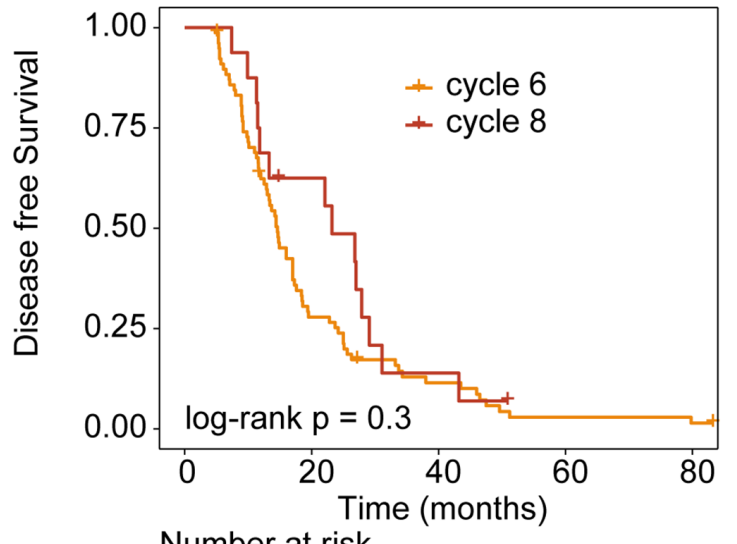

Number at risk

$\begin{array}{ccccc}\text { cycle } 6-78 & 21 & 8 & 2 & 1 \\ \text { cycle } 8-16 & 9 & 2 & 0 & 0\end{array}$

C

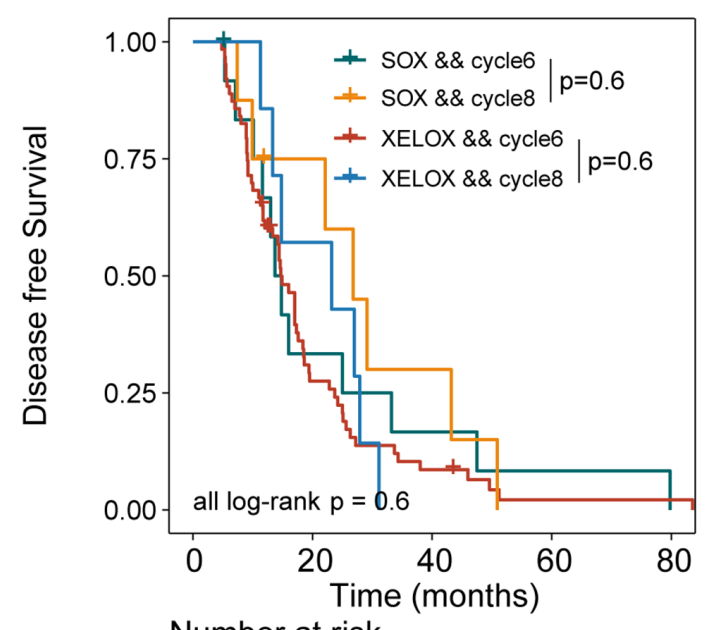

Number at risk

$\begin{array}{rcccc}\text { SOX \& \& cycle6 }-13 & 4 & 2 & 1 & 0 \\ \text { SOX \&\& cycle8 }-8 & 5 & 2 & 0 & 0 \\ \text { XELOX \& \& cycle6 }-63 & 16 & 5 & 1 & 1 \\ \text { XELOX \& \& cycle8 }-7 & 4 & 0 & 0 & 0\end{array}$

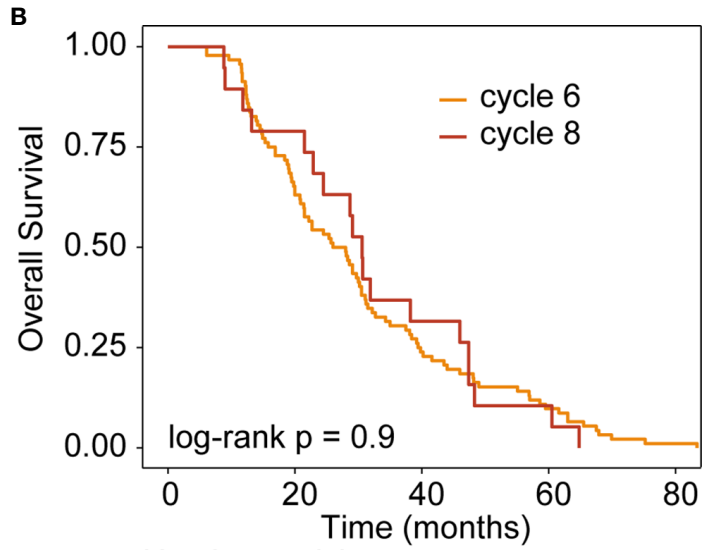

Number at risk

$\begin{array}{lllll}\text { cycle } 6+92 & 60 & 22 & 9 & 1 \\ \text { cycle } 8+19 & 15 & 6 & 2 & 0\end{array}$

D



Number at risk

$\begin{array}{rcccc}\text { SOX \& \& cycle6 }-15 & 11 & 5 & 2 & 1 \\ \text { SOX \& \& cycle8 }-8 & 6 & 2 & 0 & 0 \\ \text { XELOX \&\& cycle6 }-77 & 49 & 17 & 7 & 0 \\ \text { XELOX \& \& cycle8 }-11 & 9 & 4 & 2 & 0\end{array}$

FIGURE 3 | Kaplan-Meier survival curves for disease-free survival (DFS) and overall survival (OS). DFS (A) and OS (B) analyses for patients with stage III gastric cancer who received six or eight cycles of chemotherapy, irrespective of whether they underwent XELOX or SOX regimens. DFS (C) and OS (D) analyses for patients with stage III gastric cancer who received six or eight cycles of chemotherapy, taking the specific regimen into account. XELOX, Capecitabine plus oxaliplatin; SOX, S-1 plus oxaliplatin.

chemotherapy cycles. As far as we know, this idea was proposed for the first time. Regardless of how many cycles of chemotherapy the patient received, we did not observe significant differences between the two regimens in DFS and OS. In all subgroup analyses, only the distribution of patients classified as poorly differentiated histologically in 6 and 8 chemotherapy cycles was significantly different $(\mathrm{P}=0.034)$.

Previous prospective studies on the adjuvant treatment of GC, the ACTS-GC, CLASSIC, and ARTIST II studies, showed that compared with surgery alone, S-1, SOX and XELOX regimens have better curative effects, but these studies did not directly compare the efficacy of SOX regimen and XELOX regimen. Therefore, the difference in the efficacy of these two regimens was still unknown at that time. A single-center retrospective study showed that there was no significant difference in the efficacy of S-1 and XELOX regimens in stage III patients, but XELOX regimen was more effective than S-1 in patients with stage IIIC GC (22). Another multi-center retrospective study showed that for patients with stage IIIB or IIIC GC after D2 lymph node dissection, XELOX regimen adjuvant chemotherapy is more effective than S-1 (23). For the comparison of the effects of SOX and XELOX, a Japanese study showed that XELOX and 


\section{Cycle 6}

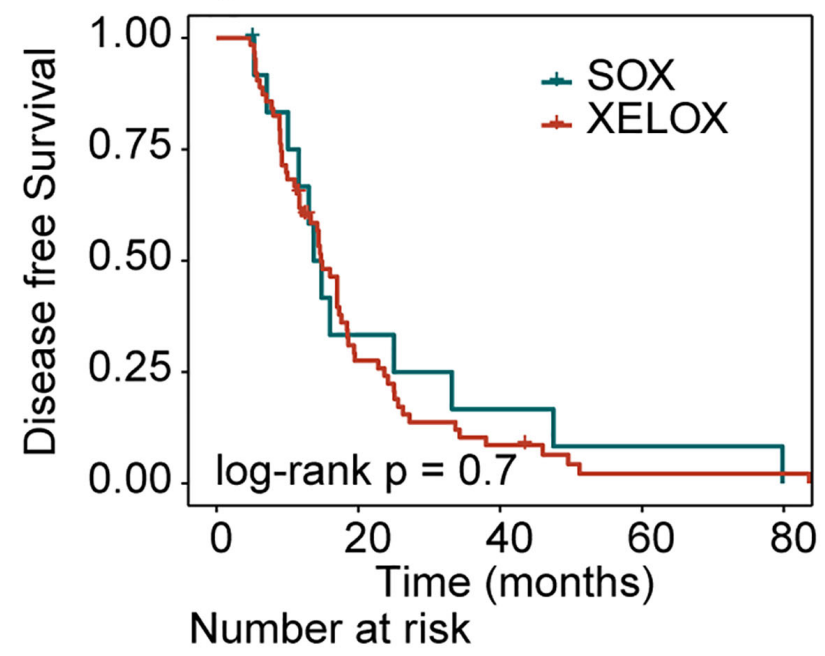

B

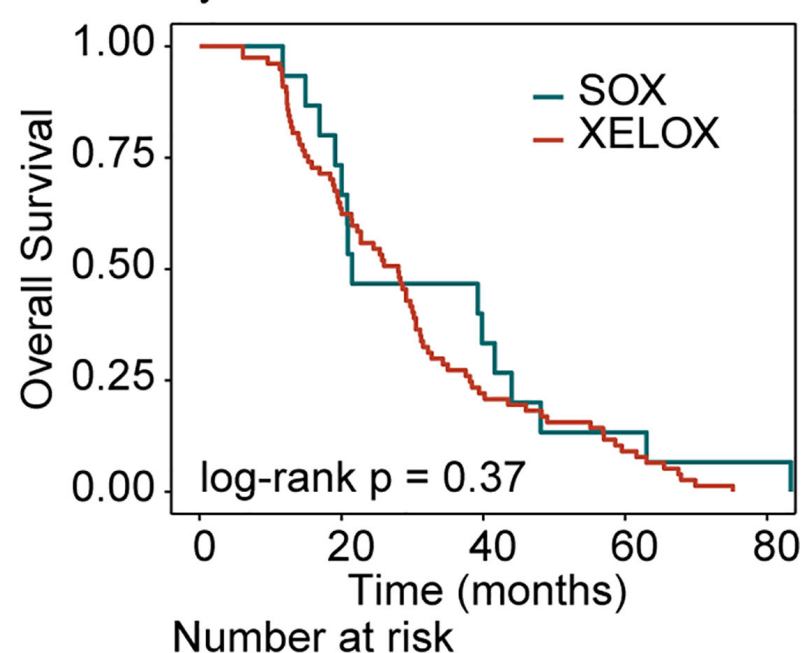

$\begin{array}{rcccc}\text { SOX }-13 & 4 & 2 & 1 & 0 \\ \text { XELOX }-63 & 16 & 5 & 1 & 1\end{array}$

C

\section{Cycle 8}

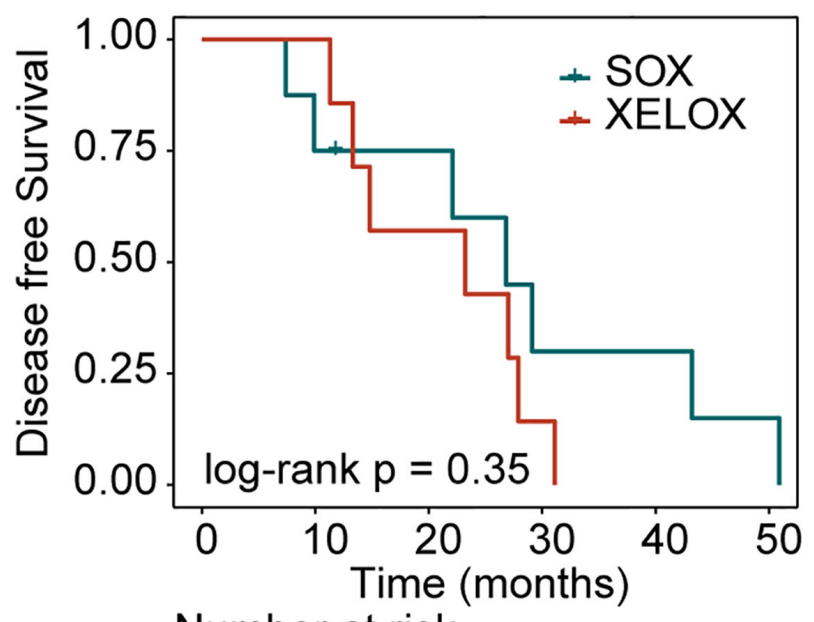

Number at risk

\begin{tabular}{r|rrrrrr} 
SOX & 8 & 6 & 5 & 2 & 2 & 1 \\
XELOX & 7 & 7 & 4 & 1 & 0 & 0
\end{tabular}

$\begin{array}{rrccc}\text { SOX }\{15 & 11 & 5 & 2 & 1 \\ \text { XELOX }-77 & 49 & 17 & 7 & 0\end{array}$

D

Cycle 8

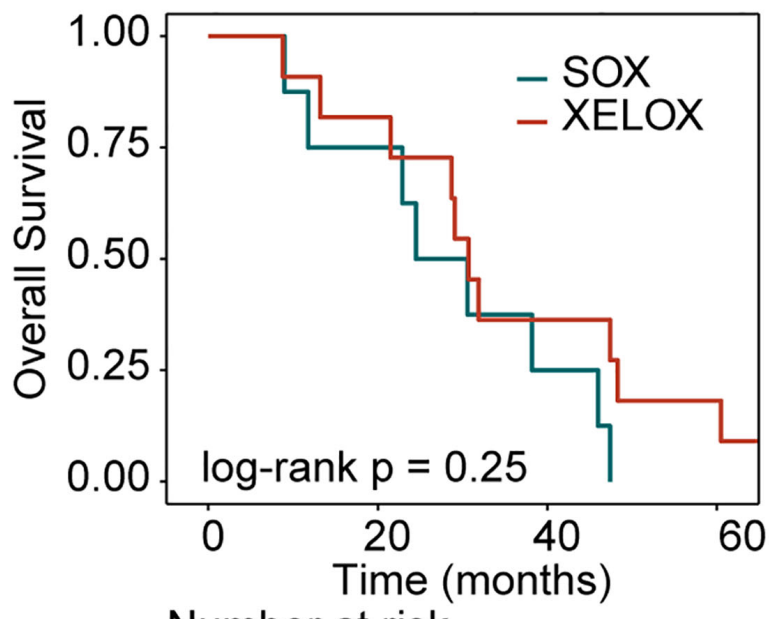

Number at risk

\begin{tabular}{|c|c|c|c|c|c|c|}
\hline sox & 8 & 7 & 6 & 4 & 2 & 0 \\
\hline XELOX & 11 & 10 & 9 & 6 & 4 & \\
\hline
\end{tabular}

FIGURE 4 | Kaplan-Meier survival curves for disease-free survival (DFS) and overall survival (OS). DFS (A) and OS (B) analyses for XELOX and SOX regimens for patients with stage III gastric cancer receiving six cycles of chemotherapy. DFS (C) and OS (D) analyses for the XELOX and SOX regimens in stage III patients receiving eight cycles of chemotherapy. XELOX, Capecitabine plus oxaliplatin; SOX, S-1 plus oxaliplatin.

SOX treatments have similar effects in patients with stage III GC who underwent D2 resection (24). Subsequently, the RESOLVE study published by ESMO in 2019 showed that the SOX adjuvant chemotherapy for 8 cycles after radical resection of GC D2 is not inferior to XELOX (25). The results of a recent single-center retrospective study also showed that SOX is as effective as XELOX for patients with GC after radical resection and that there is no significant difference in survival rate in patients receiving the different treatments (13).

The limitations of this study should be taken into consideration when analyzing the results. First, this is a singlecenter retrospective study, and the data collected will inevitably have some deviations. Second, the number of patients included is small and the sample distribution is uneven. These factors may 
A

Disease free survival (XELOX vs SOX)

\begin{tabular}{|c|c|c|c|c|}
\hline Study & Hazard Ratio & HR & $95 \%-\mathrm{Cl}$ & $\mathrm{P}$-value \\
\hline cycle_6 & & 1.01 & {$[0.54 ; 1.91]$} & 0.971 \\
\hline cycle_8 & 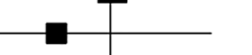 & 0.73 & {$[0.29 ; 1.82]$} & 0.494 \\
\hline & ? & & & \\
\hline
\end{tabular}

B

Overall survival (XELOX vs SOX)

\begin{tabular}{|c|c|c|c|c|}
\hline \multirow[b]{2}{*}{ Study } & \multicolumn{4}{|c|}{ ) } \\
\hline & Hazard Ratio & HR & $95 \%-\mathrm{Cl}$ & $\mathrm{P}$-value \\
\hline $\begin{array}{l}\text { cycle_6 } \\
\text { cycle_8 }\end{array}$ & $\square$ & $\begin{array}{l}1.06 \\
0.46\end{array}$ & $\begin{array}{l}{[0.61 ; 1.83]} \\
{[0.19 ; 1.12]}\end{array}$ & $\begin{array}{l}0.839 \\
0.086\end{array}$ \\
\hline & 0.51 & 5 & & \\
\hline
\end{tabular}

C

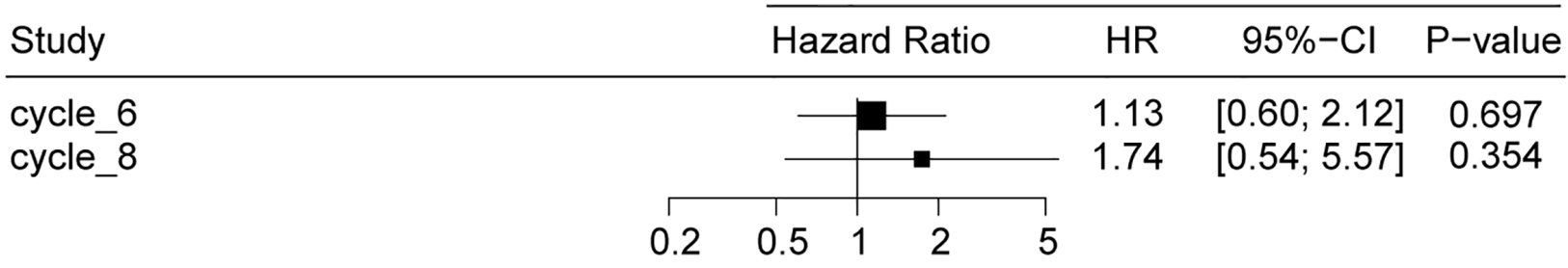

D

Overall survival (XELOX vs SOX)

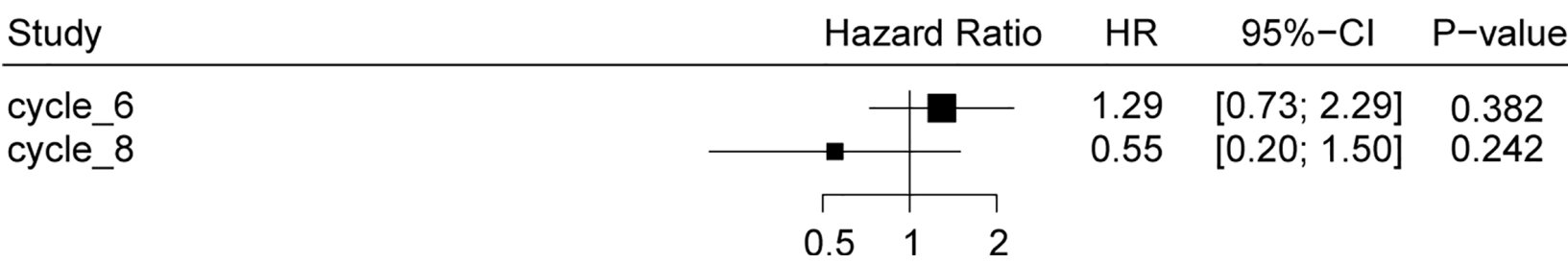

FIGURE $\mathbf{5}$ | The relationship between different chemotherapy cycles and disease-free survival (DFS) and overall survival (OS) of gastric cancer patients. DFS (A) and OS (B) analyses for patients with stage II and stage III gastric cancer. DFS (C) and OS (D) analyses for stage patients with stage III gastric cancer.

affect the experimental results. Therefore, to verify the accuracy of our results, it is necessary to conduct large-scale prospective clinical randomized controlled trials.

Together, these results and ours presented here show that the SOX chemotherapy regimen is not inferior to the XELOX regimen. Therefore, it is appropriate to compare the survival and prognosis of patients with GC receiving six and eight chemotherapy cycles, irrespective of whether they underwent SOX or XELOX regimens. Our results suggest that for patients with stage III GC, eight cycles of chemotherapy are not more effective than six cycles with regards to DFS and OS. We propose that clinically, for patients with stage III
GC, six chemotherapy cycles are effective and decrease the occurrence of chemotherapy-related adverse reactions. This result needs to be verified, but may help patients with GC choose the number of adjuvant chemotherapy cycles after surgery, avoid unnecessary increased rounds of chemotherapy, improve the quality of life, and reduce family burdens.

\section{CONCLUSION}

Six chemotherapy cycles of SOX or XELOX are as effective as eight cycles in patients with TNM stage III GC after D2 radical resection. 
A

DFS (cycle 8 vs cycle 6)

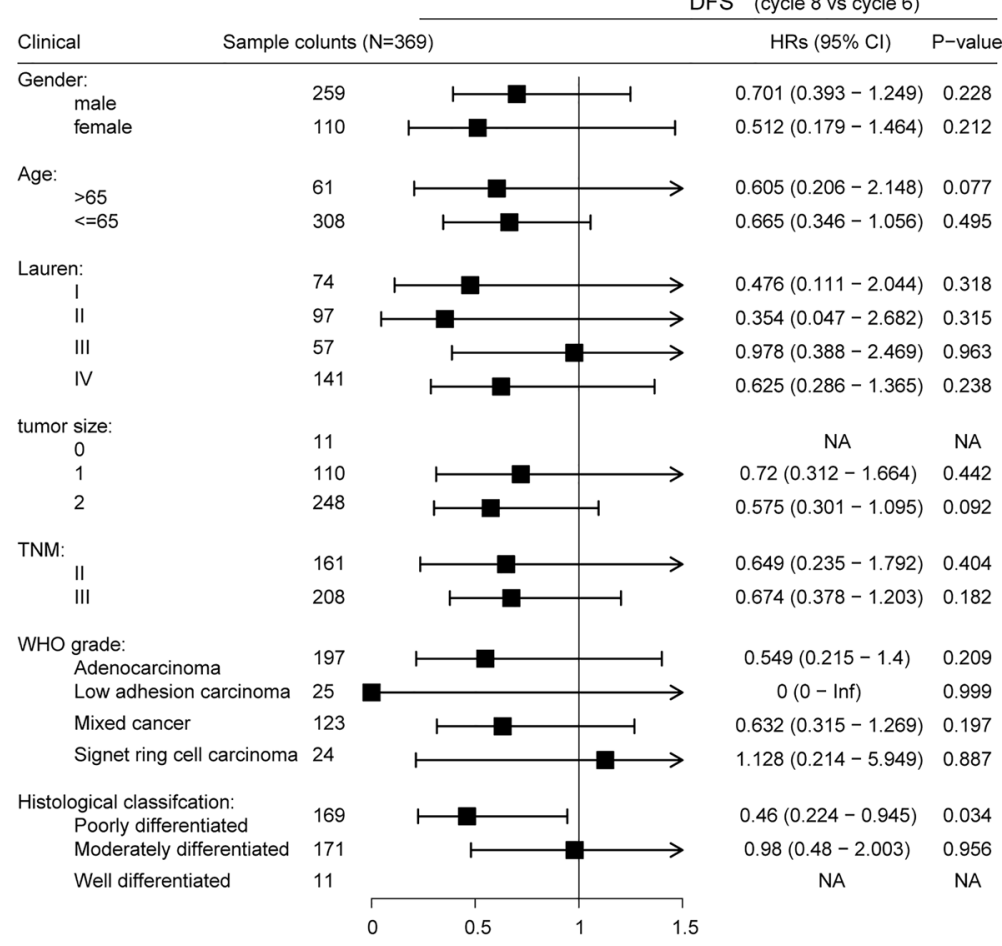

B

OS (cycle 8 vs cycle 6)

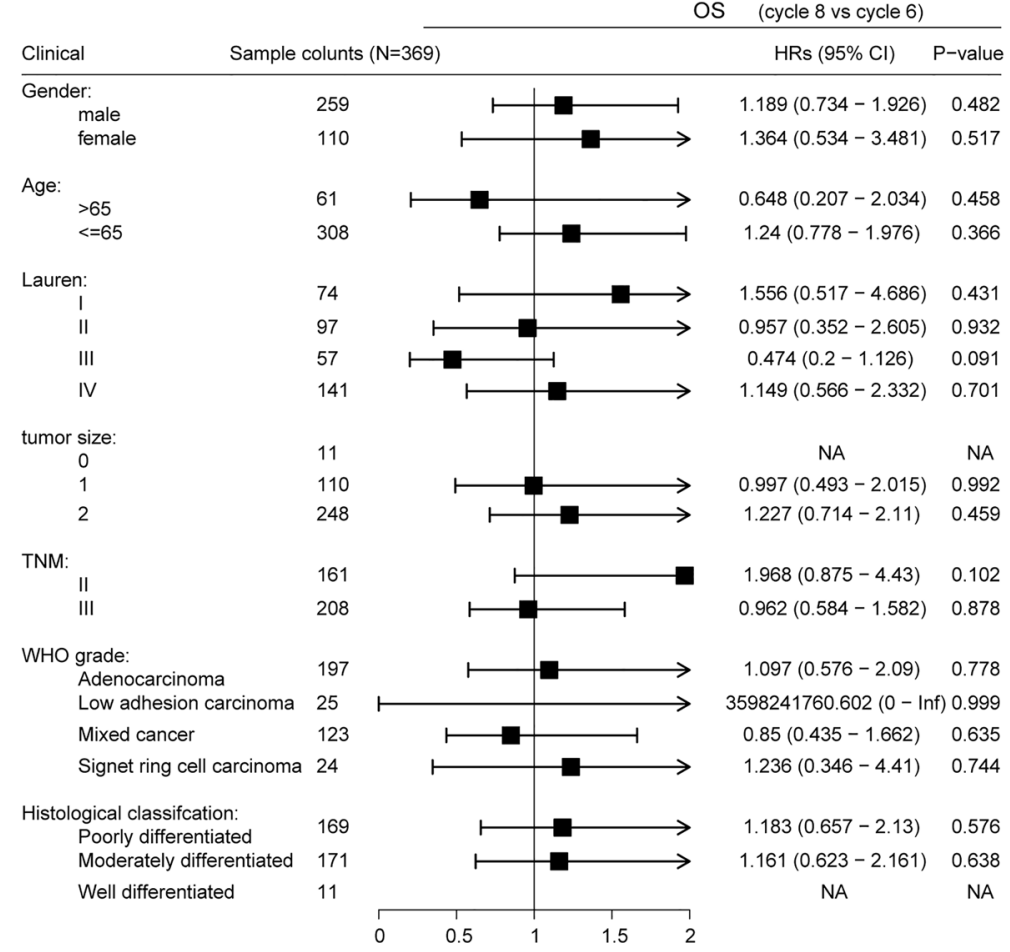

FIGURE 6 | Subgroup analyses of disease-free survival (DFS) (A) and of overall survival (OS) (B). 


\section{DATA AVAILABILITY STATEMENT}

The original contributions presented in the study are included in the article/supplementary material. Further inquiries can be directed to the corresponding authors.

\section{AUTHOR CONTRIBUTIONS}

GW and JS conceived the study. YY, ZZ and QM performed data analysis and wrote the manuscript. XF and YM provided

\section{REFERENCES}

1. Sung H, Ferlay J, Siegel RL, Laversanne M, Soerjomataram I, Jemal A, et al. Global Cancer Statistics 2020: GLOBOCAN Estimates of Incidence and Mortality Worldwide for 36 Cancers in 185 Countries. CA Cancer J Clin (2021) 0:1-41. doi: 10.3322/caac.21660

2. Chen W, Zheng R, Baade PD, Zhang S, Zeng H, Bray F, et al. Cancer Statistics in China, 2015. CA Cancer J Clin (2016) 66(2):115-32. doi: 10.3322/caac.21338

3. Allemani C, Matsuda T, Di Carlo V, Harewood R, Matz M, Nikšić M, et al. Global Surveillance of Trends in Cancer Survival 2000-14 (Concord-3): Analysis of Individual Records for 37513025 Patients Diagnosed With One of 18 Cancers From 322 Population-Based Registries in 71 Countries. Lancet (2018) 391(10125):1023-75. doi: 10.1016/s0140-6736(17)33326-3

4. Sasako M, Sakuramoto S, Katai H, Kinoshita T, Furukawa H, Yamaguchi T, et al. Five-Year Outcomes of a Randomized Phase III Trial Comparing Adjuvant Chemotherapy With S-1 Versus Surgery Alone in Stage II or III Gastric Cancer. J Clin Oncol (2011) 29(33):4387-93. doi: 10.1200/jco.2011.36.5908

5. Bang YJ, Kim YW, Yang HK, Chung HC, Noh SH. CLASSIC Trail Investigators: Adjuvant Capecitabine and Oxaliplatin for Gastric Cancer After D2 Gastrectomy (CLASSIC): A Phase 3 Open-Label, Randomized Controlled Trail. Lancet (2012) 379(9813):315-21. doi: 10.1016/S0140-6736 (11)61873-4

6. Noh SH, Park SR, Yang HK, Chung H, Chung IJ, Kim SW, et al. Adjuvant Capecitabine Plus Oxaliplatin for Gastric Cancer After D2 Gastrectomy (CLASSIC): 5-Year Follow-Up of an Open-Label, Randomised Phase 3 Trial. Lancet Oncol (2014) 15(12):1389-96. doi: 10.1016/s1470-2045(14) 70473-5

7. Yasuhiro K, Kazuhiro Y, Mitsugu K, Wataru I, Yoshihiro K, Takeshi S, et al. A Randomized Phase III Study Comparing S-1 Plus Docetaxel With S-1 Alone as a Postoperative Adjuvant Chemotherapy for Curatively Resected Stage III Gastric Cancer (JACCRO GC-07 Trial). J Clin Oncol (2018) 36(15):4007. doi: 10.1200/JCO.18.o1138

8. Lee J, Lim DH, Kim S, Park SH, Park JO, Park YS, et al. Phase III Trial Comparing Capecitabine Plus Cisplatin Versus Capecitabine Plus Cisplatin With Concurrent Capecitabine Radiotherapy in Completely Resected Gastric Cancer With D2 Lymph Node Dissection: The ARTIST Trial. J Clin Oncol (2018) 30(3):268-73. doi: 10.1200/JCO.2011.39.1953

9. Prak SH, Zang DY, Han B, Ji JH, Kim TG, Oh SY, et al. ARTIST 2:Interm Result of a Phase III Trail Involving Adjuvant Chemotherapy and/or Chemoradiotherapy After D2 Gastrectomy in Stage II/III Gastric Cancer(GC). J Clin Oncol (2019) 37(15):4001. doi: 10.1200/JCO.2019.37.15_suppl.4001

10. Park SH, Lim DH, Sohn TS, Lee J, Zang DY, Kim ST, et al. A Randomized Phase III Trial Comparing Adjuvant Single-Agent S1, s-1 With Oxaliplatin, and Postoperative Chemoradiation With S-1 and Oxaliplatin in Patients With Node-Positive Gastric Cancer After D2 Resection: The ARTIST 2 Trial. Ann Oncol (2021) 32(3):368-74. doi: 10.1016/j.annonc.2020.11.017

11. Maehara Y. S-1 in Gastric Cancer: A Comprehensive Review. Gastric Cancer (2003) 6(1):2-8. doi: 10.1007/s10120-003-0232-9

12. Lee JL, Kang YK, Kang HJ, Lee KH, Zang DY, Ryoo BY, et al. A Randomised Multicentre Phase II Trial of Capecitabine vs S-1 as First-Line Treatment in Elderly Patients With Metastatic or Recurrent Unresectable Gastric Cancer. $\mathrm{Br}$ J Cancer (2008) 99(4):584-90. doi: 10.1038/sj.bjc.6604536 technical guidance. YY and QM carried out data collection. GW finalized the research results and the final version of the manuscript. All authors contributed to the article and approved the submitted version.

\section{FUNDING}

This work was supported by grants from Haiyan Fund Key Project (JJZD2018-05).

13. Ji J, Shen L, Li Z, Zhang X, Liang H, Xue Y, et al. Perioperative Chemotherapy of Oxaliplatin Combined With S-1 (SOX) Versus Postoperative Chemotherapy of SOX or Oxaliplatin With Capecitabine (XELOX) in Locally Advanced Gastric Adenocarcinoma With D2 Gastrectomy: A Randomized Phase III Trial (RESOLVE Trial). Ann Oncol (2019) 30(5):877. doi: 10.1093/annonc/mdz394.033

14. D’Angelica M, Gonen M, Brennan MF, Turnbull AD, Bains M, Karpeh MS. Patterns of Initial Recurrence in Completely Resected Gastric Adenocarcinoma. Ann Surg (2004) 240(5):808-16. doi: 10.1097/01.sla.0000143245.28656.15

15. Gao JP, Xu W, Liu WT, Yan M, Zhu ZG. Tumor Heterogeneity of Gastric Cancer: From the Perspective of Tumorinitiating Cell. World J Gastroenterol (2018) 24:2567-81. doi: 10.3748/wjg.v24.i24.2567

16. Wu CW, Lo SS, Shen $\mathrm{KH}$, Hsieh MC, Chen JH, Chiang JH, et al. Incidence and Factors Associated With Recurrence Patterns After Intended Curative Surgery for Gastric Cancer. World J Surg (2003) 27(2):153-8. doi: 10.1007/ s00268-002-6279-7

17. Hoff PM, Fuchs CS. The Experience With Oxaliplatin in the Treatment of Upper Gastrointestinal Carcinomas. Semin Oncol (2003) 30(4):54-61. doi: 10.1016/s0093-7754(03)00406-8

18. Hong YS, Song SY, Lee SI, Chung HC, Choi SH, Noh SH, et al. A Phase II Trial of Capecitabine in Previously Untreated Patients With Advanced and/or Metastatic Gastric Cancer. Ann Oncol (2004) 15(9):1344-7. doi: 10.1093/ annonc/mdh343

19. Sumpter K, Harper-Wynne C, Cunningham D, Rao S, Tebbutt N, Norman AR, et al. Report of Two Protocol Planned Interim Analyses in a Randomised Multicentre Phase III Study Comparing Capecitabine With Fluorouracil and Oxaliplatin With Cisplatin in Patients With Advanced Oesophagogastric Cancer Receiving ECF. Br J Cancer (2005) 92(11):1976-83. doi: 10.1038/sj.bjc.6602572

20. Koizumi W, Narahara H, Hara T, Takagane A, Akiya T, Takagi M, et al. S-1 Plus Cisplatin Versus S-1 Alone for First-Line Treatment of Advanced Gastric Cancer (SPIRITS Trial): A Phase III Trial. Lancet (2008) 9(3):215-21. doi: 10.1016/S1470-2045(08)70035-4

21. Koizumi W, Takiuchi H, Yamada Y, Boku N, Fuse N, Muro K, et al. Phase II Study of Oxaliplatin Plus S-1 as First-Line Treatment for Advanced Gastric Cancer (G-SOX Study). Ann Oncol (2010) 21(5):1001-5. doi: 10.1093/ annonc/mdp464

22. Cho JH, Lim JY, Cho JY. Comparison of Capecitabine and Oxaliplatin With S-1 as Adjuvant Chemotherapy in Stage III Gastric Cancer After D2 Gastrectomy. PloS One (2017) 12(10):e0186362. doi: 10.1371/journal.pone.0186362

23. Kim IH, Park SS, Lee CM, Kim MC, Kwon IK, Min JS, et al. Efficacy of Adjuvant s-1 Versus Xelox Chemotherapy for Patients With Gastric Cancer After D2 Lymph Node Dissection: A Retrospective, Multi-Center Observational Study. Ann Surg Oncol (2018) 25(5):1176-83. doi: 10.1245/ s10434-018-6375-Z

24. Nakamura Y, Yamanaka T, Chin K, Cho H, Katai H, Terashima M, et al. Survival Outcomes of Two Phase 2 Studies of Adjuvant Chemotherapy With S-1 Plus Oxaliplatin or Capecitabine Plus Oxaliplatin for Patients With Gastric Cancer After D2 Gastrectomy. Ann Surg Oncol (2019) 26(2):46572. doi: 10.1245/s10434-018-7063-8

25. Jiang Z, Sun Y, Zhang W, Cui C, Yang L, Zhou A. Comparison of S-1 Plus Oxaliplatin (SOX) and Capecitabine Plus Oxaliplatin (XELOX) as Adjuvant Chemotherapies for Stage II and III Gastric Cancer After D2 Resection: A 
Single-Center Retrospective Study. Asia Pac J Clin Oncol (2020) 16(3):180-6. doi: 10.1111/ajco.13321

Conflict of Interest: The authors declare that the research was conducted in the absence of any commercial or financial relationships that could be construed as a potential conflict of interest.
Copyright $\odot 2021 \mathrm{Yu}$, Zhang, Meng, Ma, Fan, Sun and Wang. This is an open-access article distributed under the terms of the Creative Commons Attribution License (CC BY). The use, distribution or reproduction in other forums is permitted, provided the original author(s) and the copyright owner(s) are credited and that the original publication in this journal is cited, in accordance with accepted academic practice. No use, distribution or reproduction is permitted which does not comply with these terms. 